\title{
MULTIPLE TRAVELLING WAVE SOLUTIONS OF THREE-COMPONENT SYSTEMS WITH COMPETITION AND DIFFUSION
}

\author{
HIDEO IKEDA*
}

\begin{abstract}
Travelling wave solutions of three-component systems with competition and diffusion in which the first two species diffuse slowly but react fast than the third species are considered. Under the assumption that these systems have two stable equilibrium states, $P_{ \pm}$, the multiple existence and stability of travelling wave solutions connecting $P_{-}$with $P_{+}$are shown by using analytical singular perturbation method and the SLEP method. Velocity of a travelling wave solution surely depends on the third species. For the multiple existence of stable solutions, the dependency of its velocity on the third species is important.
\end{abstract}

1. Introduction. In this article, we study the following 3-component reactiondiffusion systems for three competing species:

$$
\left\{\begin{array}{l}
\varepsilon \tau \mathbf{u}_{t}=\varepsilon^{2} D \mathbf{u}_{x x}+\mathbf{f}(\mathbf{u}, v) \\
v_{t}=v_{x x}+g(\mathbf{u}, v)
\end{array} \quad,(t, x) \in \mathbf{R}_{+} \times \mathbf{R},\right.
$$

where $D=\operatorname{diag}\{1, d\}$ and

$$
\begin{gathered}
\mathbf{u}=\left(\begin{array}{l}
u_{1} \\
u_{2}
\end{array}\right), \mathbf{f}(\mathbf{u}, v)=\left(\begin{array}{c}
f_{1}(\mathbf{u}, v) \\
f_{2}(\mathbf{u}, v)
\end{array}\right)=\left(\begin{array}{c}
a\left(1-u_{1}-\alpha_{12} u_{2}-\alpha_{13} v\right) u_{1} \\
b\left(1-\alpha_{21} u_{1}-u_{2}-\alpha_{23} v\right) u_{2}
\end{array}\right), \\
g(\mathbf{u}, v)=\left(1-\alpha_{31} u_{1}-\alpha_{32} u_{2}-v\right) v .
\end{gathered}
$$

That is, $(\mathbf{u}, v)(t, x)$ denote the population densities of three competing species at time $t$ and spatial position $x$. Without loss of generality, it is assumed here that the intrinsic growth rates of each species are $a, b$ and 1 and the intraspecific competition rates of each one (say $\alpha_{i i}$ with $\left.i=1,2,3\right)$ are all one. $\alpha_{i j}(i \neq j)$ are the interspecific competition rates, which are positive constants. $\tau, d$ are also positive constants. Here we assume that two species $u_{1}$ and $u_{2}$ diffuse slowly but react fast relative to the third species $v$.

$$
\varepsilon>0 \text { is sufficiently small. }
$$

Null sets of the nonlinearities of $f_{1}, f_{2}$ and $g$ are given in Fig.1.1. Here we assume the following: If the $u_{1}$ species is absent, the $u_{2}$ and the $v$ species coexist. That is, we may say that if the competition between the $u_{2}$ and the $v$ species is not so strong, they can coexist. Similarly if the $u_{2}$ species is absent, the $u_{1}$ and the $v$ species coexist. Thus, we assume

$$
\alpha_{13}<1, \alpha_{23}<1, \alpha_{31}<1, \alpha_{32}<1 .
$$

Let $q_{-}=\left(1-\alpha_{21}\right) /\left(\alpha_{23}-\alpha_{21} \alpha_{13}\right)$ and $q_{+}=\left(1-\alpha_{12}\right) /\left(\alpha_{13}-\alpha_{12} \alpha_{23}\right)$ be the third components of $Q_{-}$and $Q_{+}$, and $p_{-}=\left(1-\alpha_{31}\right) /\left(1-\alpha_{13} \alpha_{31}\right)$ and $p_{+}=$ $\left(1-\alpha_{32}\right) /\left(1-\alpha_{23} \alpha_{32}\right)$ be the third components of $P_{-}$and $P_{+}$(see Fig.1.1), respectively. And we use the symbol $I(a \sim b) \equiv(a, b)$ if $a<b, \equiv\{a\}$ if $a=b, \equiv(b, a)$

*Department of Mathematics, Toyama University, Toyama 930-8555, Japan. 
if $b<a$. We assume that $1-\alpha_{13} \omega>0$ and $1-\alpha_{23} \omega>0$ for any $\omega \in I\left(p_{-} \sim p_{+}\right)$(see Fig.2.1). That is,

$$
\max \left\{p_{-}, p_{+}\right\}<\min \left\{1 / \alpha_{13}, 1 / \alpha_{23}\right\}
$$

is assumed. Furthermore we assume the one of the following three conditions depending on the relation between $\alpha_{12}, \alpha_{13}, \alpha_{21}, \alpha_{23}$ :

$$
\begin{aligned}
& I\left(p_{-} \sim p_{+}\right) \subset\left(q_{-}, q_{+}\right) \text {when } \max \left\{\alpha_{21}, 1 / \alpha_{12}\right\}<\alpha_{23} / \alpha_{13} \\
& I\left(p_{-} \sim p_{+}\right) \subset\left(q_{+}, q_{-}\right) \text {when } \alpha_{23} / \alpha_{13}<\min \left\{\alpha_{21}, 1 / \alpha_{12}\right\} \\
& \max \left\{p_{-}, p_{+}\right\}<\min \left\{q_{-}, q_{+}\right\} \text {when } 1 / \alpha_{12}<\alpha_{23} / \alpha_{13}<\alpha_{21} .
\end{aligned}
$$

The term (H4) is used in a sense that any condition of the above three is taken. Every case of (H4) guarantees both relations $1-\alpha_{13} \omega>\left(1-\alpha_{23} \omega\right) / \alpha_{21}$ and $1-\alpha_{23} \omega>\left(1-\alpha_{13} \omega\right) / \alpha_{12}$ for any $\omega \in I\left(p_{-} \sim p_{+}\right)$(see Fig.2.1).

Under the assumptions (H2), (H3) and (H4), the equilibrium states $P_{-}$and $P_{+}$ are both asymptotically stable (Fig.1.1 corresponds to the case satisfying (H2), (H3) and (H4-a)).

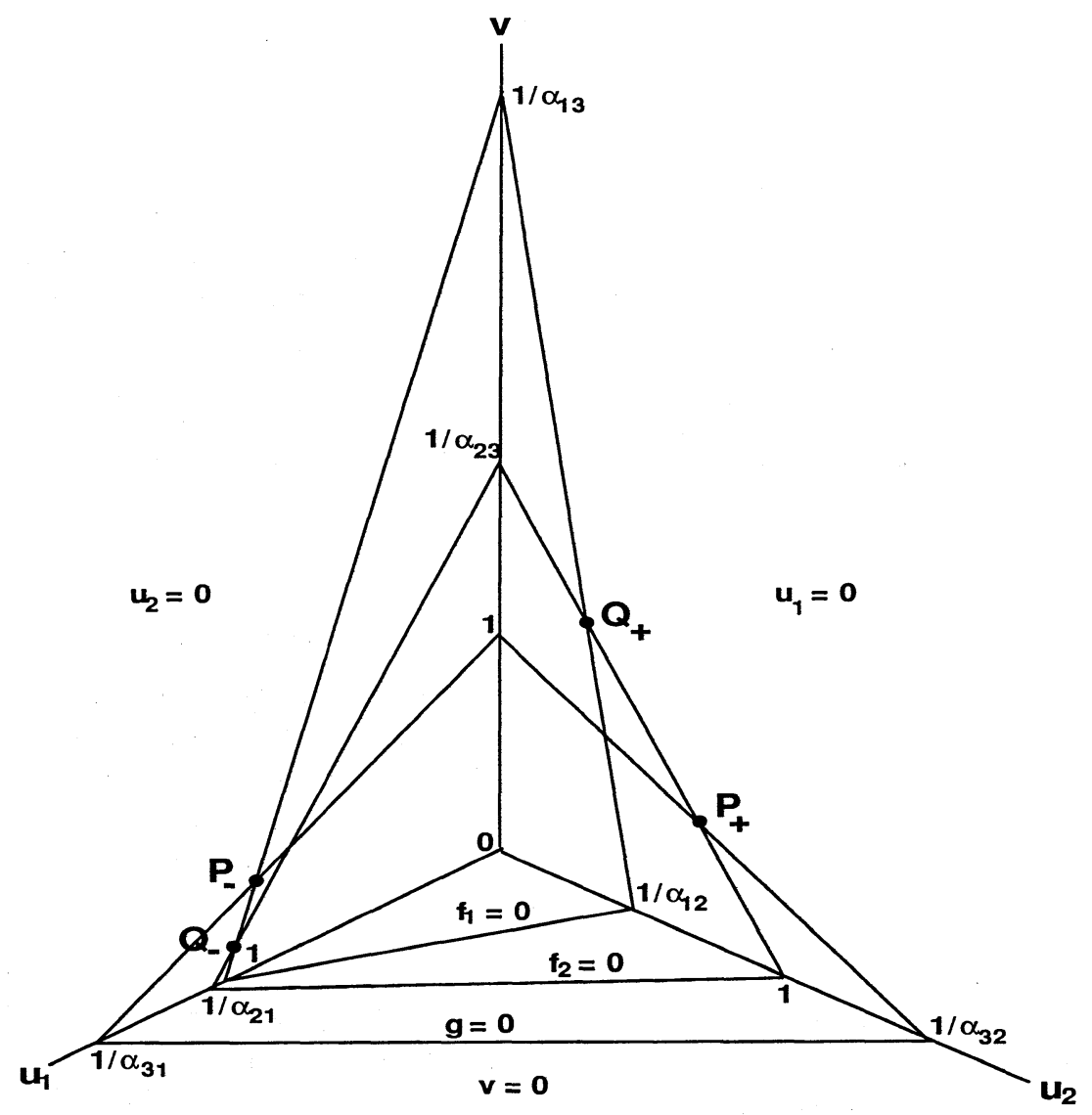

Fig. 1.1. Null sets of $f_{1}, f_{2}$ and $g$. 
This system is already discussed by Miller [M1], [M2] when $\tau=\frac{1}{\varepsilon}(\tau$ is sufficiently large). Under the same assumptions in this paper, that is, $(\mathrm{H} 1),(\mathrm{H} 2),(\mathrm{H} 3)$ and $(\mathrm{H} 4)$, he shows the existence and stability of a travelling wave solution connecting the states $P_{-}$with $P_{+}$. Furthermore it looks like unique numerically. On the other hand, we study the activator-inhibitor system in [IMN] and [NMIF], and show that the introduction of small positive parameter $\varepsilon \tau$ in fornt of $\mathbf{u}_{t}$ causes the multiple existence of stable travelling wave solutions. Keeping these situations in mind, we solve (1.1) numerically for small $\tau$. These are shown in Fig.1.2 and Fig.1.3. Fig.1.2 implies that (1.1) has two stable travelling wave solutions for small $\tau$. For the parameters in Fig.1.3, we can find only one stable travelling wave solution. That is, Fig.1.3 denies the above assertion. Then what is a difference between these two ?
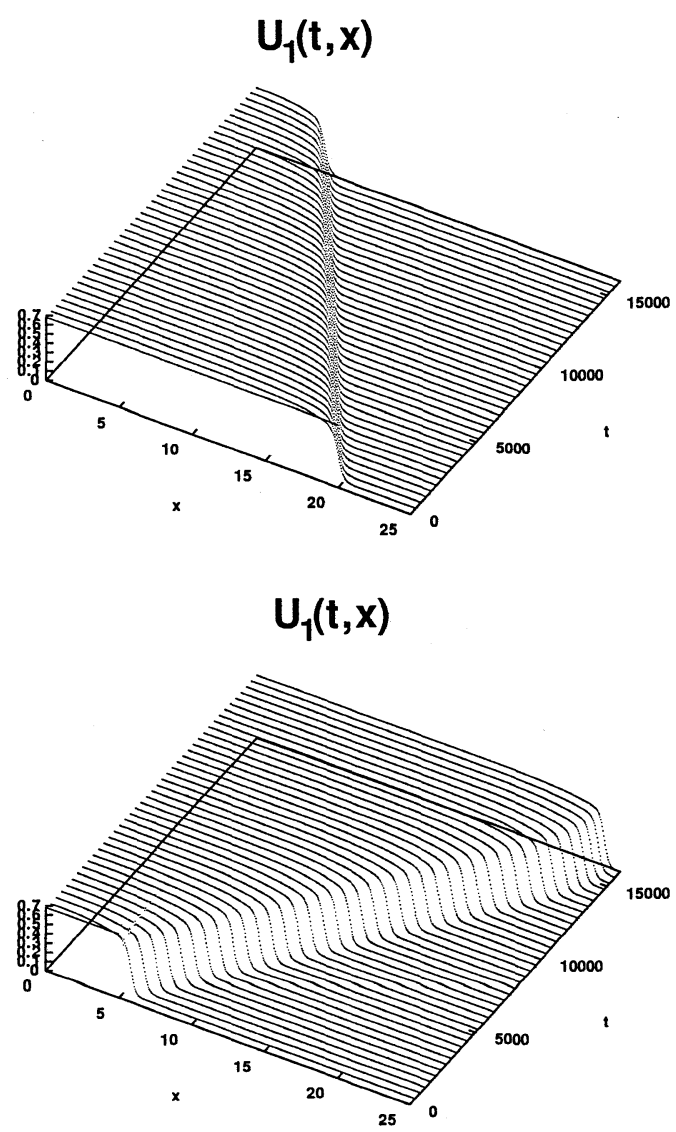

FIG. 1.2. Numerical solutions of $u_{1}$-component of (1.1) with $\varepsilon=0.01, \tau=0.1, a=b=1.0, d=$ 2.0. Two stable travelling wave solutions with $\alpha_{12}=1.3, \alpha_{13}=0.7, \alpha_{21}=0.95, \alpha_{23}=0.8, \alpha_{31}=$ $0.8, \alpha_{32}=0.5$.

This motivates us to study the existence and stability of travelling fronts connecting the states $P_{-}$with $P_{+}$rigorously. First, we should note that our system (1.1) does not fall into the category of activator-inhibitor systems. But $\mathbf{u}$ will act as an 


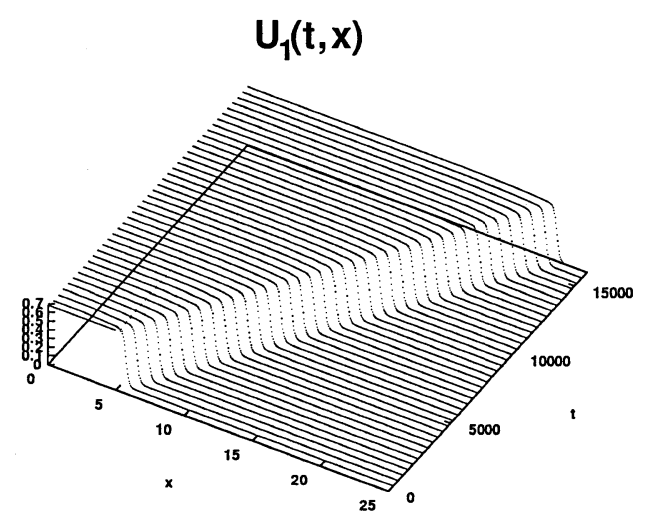

FIG. 1.3. Numerical solutions of $u_{1}$-component of (1.1) with $\varepsilon=0.01, \tau=0.1, a=b=1.0, d=$ 2.0. One stable travelling wave solution with $\alpha_{12}=0.95, \alpha_{13}=0.7, \alpha_{21}=1.5, \alpha_{23}=0.5, \alpha_{31}=$ $0.8, \alpha_{32}=0.6$.

activator and $v$ will do as an inhibitor in our analysis. Our conlusion is as follows: Let us consider the velocity of travelling wave solutions of a bistable subsystem

$$
\tau \mathbf{u}_{t}=D \mathbf{u}_{x x}+\mathbf{f}(\mathbf{u}, \omega)
$$

where $\omega$ is arbitrarily fixed in some interval. When we write its velocity by $\theta(\tau ; \omega)$, the sign of the derivative $\frac{\partial \theta}{\partial \omega}\left(\tau ; \omega_{0}\right)$ is important, where $\omega_{0}$ satisfies $\theta\left(\tau ; \omega_{0}\right)=0$. That is, its sign is negative in Fig.1.2 but that is positive in Fig.1.3. Fortunately, its sign is negative definite in the activator-inhibitor system of [IMN]. These basic study will be important for pattern formation in higher dimensional spaces.

We use the following function spaces. Let $\varepsilon$ and $\sigma$ be positive numbers, $0<s<1$ and $n$ be an nonnegative integer. Let

$$
\begin{aligned}
& X_{\sigma, \varepsilon}^{n}\left(\mathbf{R}_{ \pm}\right) \equiv\left\{u \in C^{n}\left(\mathbf{R}_{ \pm}\right)\left|\|u\|_{X_{\sigma, \varepsilon}^{n}\left(\mathbf{R}_{ \pm}\right)}=\sum_{i=0}^{n} \sup _{x \in \mathbf{R}_{ \pm}} e^{\sigma|x|}\right|\left(\varepsilon \frac{d}{d x}\right)^{i} u(x) \mid<\infty\right\}, \\
& \stackrel{\circ}{\sigma, \varepsilon}_{\sigma, \varepsilon}\left(\mathbf{R}_{ \pm}\right) \equiv\left\{u \in X_{\sigma, \varepsilon}^{n}\left(\mathbf{R}_{ \pm}\right) \mid u(0)=0\right\}, \quad X_{\sigma, \varepsilon}^{n}(\mathbf{R}) \equiv X_{\sigma, \varepsilon}^{n}\left(\mathbf{R}_{-}\right) \cup X_{\sigma, \varepsilon}^{n}\left(\mathbf{R}_{+}\right), \\
& H^{s}(\mathbf{R}) \equiv \text { the interpolation space }\left[H^{1}(\mathbf{R}), L^{2}(\mathbf{R})\right]_{1-s},
\end{aligned}
$$

$B C(\mathbf{R}) \equiv$ the set of bounded and uniformly continuous functions defined on $\mathbf{R}$, $\left(H^{n}\right)^{\sharp}(\mathbf{R}) \equiv$ the dual space of $H^{n}(\mathbf{R}), \quad\left(H^{s}\right)^{\sharp}(\mathbf{R}) \equiv$ the dual space of $H^{s}(\mathbf{R})$. 
2. Travelling Front Solutions. Introducing the travelling coordinate $z=x+$ $\theta t$, we see that travelling front solutions with velocity $\theta$ satisfy

$$
\left\{\begin{array}{c}
\varepsilon^{2} D \mathbf{u}_{z z}-\varepsilon \tau \theta \mathbf{u}_{z}+\mathbf{f}(\mathbf{u}, v)=\mathbf{0} \\
v_{z z}-\theta v_{z}+g(\mathbf{u}, v)=0 \\
(\mathbf{u}, v)(-\infty)=P_{-},(\mathbf{u}, v)(+\infty)=P_{+} .
\end{array}, z \in\right.
$$

To avoid the phase ambiguity, we impose the condition on $u_{1}(z)$ :

$$
u_{1}(0)=\beta
$$

where $\beta$ is an arbitrarily fixed value in some interval. Moreover, we put

$$
\left(u_{2}, v\right)(0)=(\nu, \omega)
$$

for $\nu$ and $\omega$, which will be determined later.

We devide the whole interval $\mathbf{R}$ into two subintervals $\mathbf{R}_{-}$and $\mathbf{R}_{+}$. First, fix $\theta$, $\nu$ and $\omega$ arbitrarily, and look for solutions $\left(\mathbf{u}^{ \pm}, v^{ \pm}\right)=\left(u_{1}^{ \pm}, u_{2}^{ \pm}, v^{ \pm}\right)$of the following boundary value problem on each subinterval $\mathbf{R}_{ \pm}$with the aid of outer and inner approximations:

$$
\left\{\begin{array}{l}
\varepsilon^{2} D \mathbf{u}_{z z}^{ \pm}-\varepsilon \tau \theta \mathbf{u}_{z}^{ \pm}+\mathbf{f}\left(\mathbf{u}^{ \pm}, v^{ \pm}\right)=\mathbf{0} \\
v_{z z}^{ \pm}-\theta v_{z}^{ \pm}+g\left(\mathbf{u}^{ \pm}, v^{ \pm}\right)=0 \\
\left(\mathbf{u}^{ \pm}, v^{ \pm}\right)(0)=(\beta, \nu, \omega) \\
\left(\mathbf{u}^{-}, v^{-}\right)(-\infty)=P_{-},\left(\mathbf{u}^{+}, v^{+}\right)(+\infty)=P_{+} .
\end{array}, z \in \mathbf{R}_{ \pm}\right.
$$

Second, we derive three relations between $\theta, \nu$ and $\omega$ through $C^{1}$-matching of the solutions of $(2.4)_{ \pm}$at $z=0$, and solve these relations to obtain $\theta=\theta(\tau ; \varepsilon), \nu=\nu(\tau ; \varepsilon)$ and $\omega=\omega(\tau ; \varepsilon)$.

2.1. Outer Approximations. We assume that $z=0$ is only the layer position of solutions. This implies that the derivatives of $\mathbf{u}^{ \pm}$are moderate in the region away from $z=0$. Therefore, solutions of the following limiting equations of $(2.4)_{ \pm}$as $\varepsilon \downarrow 0$ could become good approximations there:

$$
\left\{\begin{array}{l}
\mathbf{f}\left(\mathbf{u}^{ \pm}, v^{ \pm}\right)=\mathbf{0} \\
v_{z z}^{ \pm}-\theta v_{z}^{ \pm}+g\left(\mathbf{u}^{ \pm}, v^{ \pm}\right)=0 \quad, z \in \mathbf{R}_{ \pm} \\
v^{-}(-\infty)=p_{-}, v^{ \pm}(0)=\omega, v^{+}(+\infty)=p_{+}
\end{array}\right.
$$

If $p_{-}=p_{+},(2.5)_{ \pm}$have trivial solutions $v^{ \pm}(z)=p_{-}$with $\omega=p_{-}$. If $p_{-} \neq p_{+}$, we choose $\omega$ arbitrarily in the interval $I\left(p_{-} \sim p_{+}\right)$and fix it. Hereafter we assume $p_{-}<$ $p_{+}$because the other case is treated similarly. As particular solutions of $\mathbf{f}\left(\mathbf{u}^{ \pm}, v^{ \pm}\right)=\mathbf{0}$, we take the following relations

$$
\left\{\begin{array}{l}
\mathbf{u}^{-}=\left(u_{1}^{-}, u_{2}^{-}\right)=\left(1-\alpha_{13} v^{-}, 0\right) \\
\mathbf{u}^{+}=\left(u_{1}^{+}, u_{2}^{+}\right)=\left(0,1-\alpha_{23} v^{+}\right)
\end{array}\right.
$$


Substituting these into the second equations, we find that $(2.5)_{ \pm}$are reduced to

$$
\left\{\begin{array}{l}
V_{z z}^{-}-\theta V_{z}^{-}+g\left(1-\alpha_{13} V^{-}, 0, V^{-}\right)=0, \quad z \in \mathbf{R}_{-} \\
V_{z z}^{+}-\theta V_{z}^{+}+g\left(0,1-\alpha_{23} V^{+}, V^{+}\right)=0, \quad z \in \mathbf{R}_{+} \\
V^{-}(-\infty)=p_{-}, V^{ \pm}(0)=\omega, V^{+}(+\infty)=p_{+} .
\end{array}\right.
$$

For this equation, we have the following lemma:

Lemma 2.1.([IMN, Lemma 2.1]) For any fixed $\theta \in \mathbf{R}$ and $\omega \in\left(p_{-}, p_{+}\right),(2.6)_{ \pm}$ have unique monotone increasing solutions $V^{ \pm}(z ; \theta, \omega)$ satisfying

$$
\left|V^{ \pm}(z ; \theta, \omega)-p_{ \pm}\right| \in X_{\sigma_{0}, 1}^{2}\left(\mathbf{R}_{ \pm}\right),
$$

where $\sigma_{0}=\min \left\{\sigma_{-}, \sigma_{+}\right\}, \sigma_{-}=\sqrt{1-\alpha_{31}}$ and $\sigma_{+}=\sqrt{1-\alpha_{32}}$. Moreover they satisfy (i) $V^{ \pm}(z ; \theta, \omega)$ are uniformly continuous with respect to $(\theta, \omega) \in \mathbf{R} \times\left(p_{-}, p_{+}\right)$in the $X_{\sigma_{0}, 1}^{2}\left(\mathbf{R}_{ \pm}\right)$-topology,

(ii) $\frac{\partial}{\partial \theta}\left[\frac{d}{d z} V^{-}(0 ; \theta, \omega)-\frac{d}{d z} V^{+}(0 ; \theta, \omega)\right]>0$,

(iii) $\frac{\partial}{\partial \omega}\left[\frac{d}{d z} V^{-}(0 ; \theta, \omega)-\frac{d}{d z} V^{+}(0 ; \theta, \omega)\right]>0$.

By this lemma, we directly obtain the following relation which is important for the existence and stability of travelling wave solutions.

LEMma 2.2. For any fixed $\theta \in \mathbf{R}$, there uniquely exists $\omega=\omega_{O}(\theta)$ satisfying

$$
\frac{d}{d z} V^{-}\left(0 ; \theta, \omega_{O}(\theta)\right)-\frac{d}{d z} V^{+}\left(0 ; \theta, \omega_{O}(\theta)\right)=0,
$$

which is a strictly monotone decreasing function of $\theta \in \mathbf{R}$ and converges to $p_{ \pm}$as $\theta \rightarrow \mp \infty$, respectively.

Define $\mathbf{U}^{ \pm}(z ; \theta, \omega)$ by

$$
\begin{cases}\mathbf{U}^{-}(z ; \theta, \omega)=\left(U_{1}^{-}, U_{2}^{-}\right)(z ; \theta, \omega)=\left(1-\alpha_{13} V^{-}(z ; \theta, \omega), 0\right) & \text { for } x \in \mathbf{R}_{-} \\ \mathbf{U}^{+}(z ; \theta, \omega)=\left(U_{1}^{+}, U_{2}^{+}\right)(z ; \theta, \omega)=\left(0,1-\alpha_{23} V^{+}(z ; \theta, \omega)\right) & \text { for } x \in \mathbf{R}_{+} .\end{cases}
$$

We have outer approximations of $(2.4)_{ \pm}$as $\left(\mathbf{U}^{ \pm}, V^{ \pm}\right)(z ; \theta, \omega)$.

2.2. Inner Approximations. Since the outer approximations $\mathbf{U}^{ \pm}(z ; \theta, \omega)$ do not satisfy the bounbary conditions at $z=0$, we must remedy them in a neighborhood of $z=0$. For this purpose, it is convenient to introduce the stretched variable $\xi=z / \varepsilon$. Substituting $\left(\mathbf{U}^{ \pm}+\overline{\mathbf{u}}^{ \pm}, V^{ \pm}\right)$into $(2.4)_{ \pm}$with remedy terms $\overline{\mathbf{u}}^{ \pm}$, and putting $\varepsilon=0$, we have the following problems for $\overline{\mathbf{u}}^{ \pm}(\xi)=\left(\bar{u}_{1}^{ \pm}, \bar{u}_{2}^{ \pm}\right)(\xi)$ :

$$
\left\{\begin{array}{l}
D \overline{\mathbf{u}}_{\xi \xi}^{ \pm}-\tau \theta \overline{\mathbf{u}}_{\xi}^{ \pm}+\mathbf{f}\left(\mathbf{U}^{ \pm}(0 ; \theta, \omega)+\overline{\mathbf{u}}^{ \pm}, \omega\right)=\mathbf{0} \quad, \xi \in \mathbf{R}_{ \pm} \\
\overline{\mathbf{u}}^{ \pm}(0)=(\beta, \nu)-\mathbf{U}^{ \pm}(0 ; \theta, \omega) \\
\overline{\mathbf{u}}^{ \pm}( \pm \infty)=\mathbf{0} .
\end{array}\right.
$$


That is, the inner approximations are stretched on the half lines $\mathbf{R}_{ \pm}$. First, we consider the problem on the whole line.

$$
\left\{\begin{array}{l}
D \overline{\mathbf{u}}_{\xi \xi}-\Theta \overline{\mathbf{u}}_{\xi}+\mathbf{f}(\overline{\mathbf{u}}, \omega)=\mathbf{0} \quad, \xi \in \mathbf{R} \\
\bar{u}_{1}(0)=\beta \\
\overline{\mathbf{u}}(-\infty)=\left(1-\alpha_{13} \omega, 0\right), \overline{\mathbf{u}}(+\infty)=\left(0,1-\alpha_{23} \omega\right) .
\end{array}\right.
$$

Fix $\beta \in\left(0,1-\alpha_{13} \omega\right)$ arbitrarily. The assumptions (H3) and (H4) imply that (2.8) is the bistable system (see Fig.2.1). Then we have the following lemma:

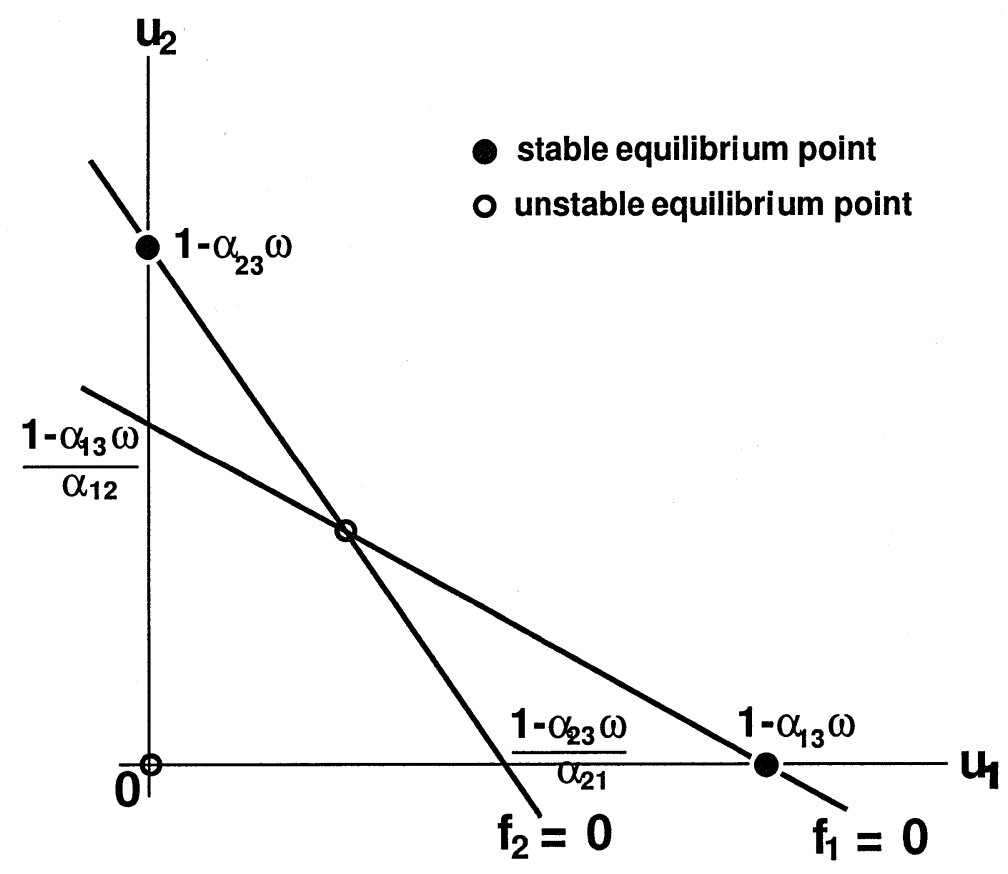

FIG. 2.1. Isoclines of $f_{1}\left(u_{1}, u_{2}, \omega\right)=0$ and $f_{2}\left(u_{1}, u_{2}, \omega\right)=0$.

LEMma 2.3.([K, Theorem 2.1]) For any fixed $\omega \in\left(p_{-}, p_{+}\right)$, there exists $\Theta$ $=\bar{\Theta}(\omega)$ such that $(2.8)$ has a strictly monotone solution $\overline{\mathbf{u}}(\xi ; \omega)=\left(\bar{u}_{1}, \bar{u}_{2}\right)(\xi ; \omega)$ with $\bar{u}_{1, \xi}(\xi ; \omega)<0, \bar{u}_{2, \xi}(\xi ; \omega)>0 . \quad \overline{\mathbf{u}}(\xi ; \omega)-\mathbf{U}^{ \pm}(0 ; \bar{\Theta}(\omega), \omega) \in\left(X_{\tau_{0}, 1}^{2}\left(\mathbf{R}_{ \pm}\right)\right)^{2}$ for some $\tau_{0}>0$.

Define $\theta_{I}(\tau ; \omega)$ by $\theta_{I}(\tau ; \omega)=\bar{\Theta}(\omega) / \tau$ for any $\omega \in\left(p_{-}, p_{+}\right)$. When we define the linearized operator of $(2.8)$ around $\overline{\mathbf{u}}(\xi ; \omega)$ by

$$
L(\mathbf{p}) \equiv D \mathbf{p}_{\xi \xi}-\bar{\Theta} \mathbf{p}_{\xi}+\overline{\mathbf{f}}_{\mathbf{u}} \mathbf{p}
$$

we know that $L\left(\overline{\mathbf{u}}_{\xi}\right)=\mathbf{0}$. The adjoint operator of $L$, say $L^{*}$, is defined by

$$
L^{*}(\mathbf{p}) \equiv D \mathbf{p}_{\xi \xi}+\bar{\Theta} \mathbf{p}_{\xi}+{ }^{t} \overline{\mathbf{f}}_{\mathbf{u}} \mathbf{p}
$$


where ${ }^{t} A$ stands for the transpose of a matrix $A$. For this operator, $[\mathrm{KY}]$ showed that any non-trivial bounded solution $\mathbf{p}^{*}(\xi)=\left(p_{1}^{*}, p_{2}^{*}\right)(\xi)$ of $L^{*}(\mathbf{p})=\mathbf{0}$ satisfies $p_{1}^{*}(\xi) p_{2}^{*}(\xi)<0$ for all $\xi \in \mathbf{R}$.

LEMMA 2.4.([I, Lemma 2.5]) $\quad \bar{\Theta}(\omega)$ is a smooth function and satisfies

$$
\begin{aligned}
\frac{\partial \bar{\Theta}}{\partial \omega}(\omega) & =-\frac{\int_{-\infty}^{\infty}\left\{a \alpha_{13} \bar{u}_{1} p_{1}^{*}+b \alpha_{23} \bar{u}_{2} p_{2}^{*}\right\} d \xi}{\int_{-\infty}^{\infty}\left\{\bar{u}_{1, \xi} p_{1}^{*}+\bar{u}_{2, \xi} p_{2}^{*}\right\} d \xi} \\
& =-\frac{\alpha_{13}}{2\left(1-\alpha_{13} \omega\right)} \bar{\Theta}(\omega)+\frac{b}{\sqrt{a}} \frac{\left(\alpha_{13}-\alpha_{23}\right)}{\left(1-\alpha_{13} \omega\right)^{3 / 2}} S,
\end{aligned}
$$

where $S>0$.

Here we arbitrarily fix $\omega^{*} \in\left(p_{-}, p_{+}\right)$and define $\theta^{*}(\tau)$ and $\nu^{*} \in\left(0,1-\alpha_{23} \omega^{*}\right)$ by $\theta^{*}(\tau)=\theta_{I}\left(\tau ; \omega^{*}\right)$ and $\bar{u}_{2}\left(0 ; \omega^{*}\right)=\nu^{*}$, respectively.

LEMma 2.5.([I, Lemma 3.2]) There exists $c_{0}>0$ such that for any $(\theta, \omega, \nu)$ satisfying $\left|\theta-\theta^{*}(\tau)\right|+\left|\omega-\omega^{*}\right|+\left|\nu-\nu^{*}\right|<c_{0},(2.7)_{ \pm}$have unique strictly monotone solutions $\overline{\mathbf{u}}^{ \pm}(\xi ; \tau ; \theta, \omega, \nu)=\left(\bar{u}_{1}^{ \pm}, \bar{u}_{2}^{ \pm}\right)(\xi ; \tau ; \theta, \omega, \nu) \in\left(X_{\tau_{0}, 1}^{2}\left(\mathbf{R}_{ \pm}\right)\right)^{2}$ such that

(i) $\overline{\mathbf{u}}^{ \pm}(\xi ; \tau ; \theta, \omega, \nu)$ are uniformly continuous with respect to $(\theta, \omega, \nu)$ in the $\left(X_{\tau_{0}, 1}^{2}\left(\mathbf{R}_{ \pm}\right)\right)^{2}$-topology

(ii) $\overline{\mathbf{u}}_{\xi}^{-}\left(0 ; \tau ; \theta^{*}(\tau), \omega^{*}, \nu^{*}\right)-\overline{\mathbf{u}}_{\xi}^{+}\left(0 ; \tau ; \theta^{*}(\tau), \omega^{*}, \nu^{*}\right)=0$,

(iii) $\left\{\frac{\partial}{\partial \theta} \bar{u}_{1, \xi}^{-}\left(0 ; \tau ; \theta^{*}(\tau), \omega^{*}, \nu^{*}\right)-\frac{\partial}{\partial \theta} \bar{u}_{1, \xi}^{+}\left(0 ; \tau ; \theta^{*}(\tau), \omega^{*}, \nu^{*}\right)\right\} p_{1}^{*}(0)$

$\left.+d\left\{\frac{\partial}{\partial \theta} \bar{u}_{2, \xi}^{-}\left(0 ; \tau ; \theta^{*}(\tau), \omega^{*}, \nu^{*}\right)-\frac{\partial}{\partial \theta} \bar{u}_{2, \xi}^{+}\left(0 ; \tau ; \theta^{*}(\tau), \omega^{*}, \nu^{*}\right)\right)\right\} p_{2}^{*}(0)$

$=\tau \int_{-\infty}^{\infty}\left\{\bar{u}_{1, \xi}(\xi) p_{1}^{*}(\xi)+\bar{u}_{2, \xi}(\xi) p_{2}^{*}(\xi)\right\} d \xi$,

(iv) $\left\{\frac{\partial}{\partial \omega} \bar{u}_{1, \xi}^{-}\left(0 ; \tau ; \theta^{*}(\tau), \omega^{*}, \nu^{*}\right)-\frac{\partial}{\partial \omega} \bar{u}_{1, \xi}^{+}\left(0 ; \tau ; \theta^{*}(\tau), \omega^{*}, \nu^{*}\right)\right\} p_{1}^{*}(0)$

$\left.+d\left\{\frac{\partial}{\partial \omega} \bar{u}_{2, \xi}^{-}\left(0 ; \tau ; \omega^{*}, \theta^{*}(\tau), \nu^{*}\right)-\frac{\partial}{\partial \omega} \bar{u}_{2, \xi}^{+}\left(0 ; \tau ; \omega^{*}, \theta^{*}(\tau), \nu^{*}\right)\right)\right\} p_{2}^{*}(0)$

$=\int_{-\infty}^{\infty}\left\{a \alpha_{13} \bar{u}_{1}(\xi) p_{1}^{*}(\xi)+b \alpha_{23} \bar{u}_{2}(\xi) p_{2}^{*}(\xi)\right\} d \xi$,

(v) $\left\{\frac{\partial}{\partial \nu} \bar{u}_{1, \xi}^{-}\left(0 ; \tau ; \theta^{*}(\tau), \omega^{*}, \nu^{*}\right)-\frac{\partial}{\partial \nu} \bar{u}_{1, \xi}^{+}\left(0 ; \tau ; \theta^{*}(\tau), \omega^{*}, \nu^{*}\right)\right\} p_{1}^{*}(0)$

$\left.+d\left\{\frac{\partial}{\partial \nu} \bar{u}_{2, \xi}^{-}\left(0 ; \tau ; \theta^{*}(\tau), \omega^{*}, \nu^{*}\right)-\frac{\partial}{\partial \nu} \bar{u}_{2, \xi}^{+}\left(0 ; \tau ; \theta^{*}(\tau), \omega^{*}, \nu^{*}\right)\right)\right\} p_{2}^{*}(0)$

$=0$.

2.3. Solutions of $(2.4)_{ \pm}$on $\mathbf{R}_{ \pm}$. In this subsection, we show the existence of solutions of $(2.4)_{ \pm}$. Fix $c_{0}$ to be positive and small, and put $\Sigma_{c_{0}} \equiv\{\rho=(\theta, \omega, \nu) \| \theta-$ $\left.\theta^{*}(\tau)|+| \omega-\omega^{*}|+| \nu-\nu^{*} \mid<c_{0}\right\}$. For any $\rho=(\theta, \omega, \nu) \in \Sigma_{c_{0}}$, define

$$
\left\{\begin{array}{l}
\mathbf{u}^{ \pm, a}(z ; \tau ; \rho)=\mathbf{U}^{ \pm}(z ; \theta, \omega)+\overline{\mathbf{u}}^{ \pm}\left(\frac{z}{\varepsilon} ; \tau ; \rho\right) \\
v^{ \pm, a}(z ; \tau ; \rho)=V^{ \pm}(z ; \theta, \omega)+\varepsilon^{2}\left\{Y^{ \pm}\left(\frac{z}{\varepsilon} ; \tau ; \rho\right)-e^{-\sigma_{0}|z|} Y^{ \pm}(0 ; \tau ; \rho)\right\}
\end{array} \quad, z \in \mathbf{R}_{ \pm}\right.
$$

where $Y^{ \pm}(\xi ; \tau ; \rho)=-\int_{ \pm \infty}^{\xi} \int_{ \pm \infty}^{t}\left\{g\left(\mathbf{U}^{ \pm}(0 ; \theta, \omega)+\overline{\mathbf{u}}^{ \pm}(s ; \tau ; \rho), \omega\right)-\right.$ $\left.g\left(\mathbf{U}^{ \pm}(0 ; \theta, \omega), \omega\right)\right\} d s d t$. We will find that $\left(\mathbf{u}^{ \pm, a}, v^{ \pm, a}\right)(z ; \tau ; \rho)$ are good approximate solutions of $(2.4)_{ \pm}$. Therefore we seek exact solutions of $(2.4)_{ \pm}$with the forms

$$
\left\{\begin{array}{l}
\mathbf{u}^{ \pm}(z ; \tau ; \varepsilon, \rho)=\mathbf{u}^{ \pm, a}(z ; \tau ; \rho)+\mathbf{r}^{ \pm}(z ; \tau ; \varepsilon, \rho)-\mathbf{b}^{ \pm} s^{ \pm}(z ; \tau ; \varepsilon, \rho) \\
v^{ \pm}(z ; \tau ; \varepsilon, \rho)=v^{ \pm, a}(z ; \tau ; \rho)+s^{ \pm}(z ; \tau ; \varepsilon, \rho)
\end{array} \quad, z \in \mathbf{R}_{ \pm},\right.
$$


where $\mathbf{r}^{ \pm}(z ; \tau ; \varepsilon, \rho)={ }^{t}\left(r_{1}^{ \pm}, r_{2}^{ \pm}\right)(z ; \tau ; \varepsilon, \rho), \mathbf{b}^{-}={ }^{t}\left(\alpha_{13}, 0\right)$ and $\mathbf{b}^{+}={ }^{t}\left(0, \alpha_{23}\right)$. Fix $\tau>0$ arbitrarily. Substituting $(2.9)_{ \pm}$into $(2.4)_{ \pm}$, we define the following operators for $\left(\mathrm{r}^{ \pm}, s^{ \pm}\right)$:

$$
\mathbf{T}^{ \pm}\left(\mathbf{r}^{ \pm}, s^{ \pm} ; \varepsilon, \rho\right) \equiv\left(\begin{array}{c}
\varepsilon^{2} D \mathbf{u}_{z z}^{ \pm}-\varepsilon \tau \theta \mathbf{u}_{z}^{ \pm}+\mathbf{f}\left(\mathbf{u}^{ \pm}, v^{ \pm}\right) \\
v_{z z}^{ \pm}-\theta v_{z}^{ \pm}+g\left(\mathbf{u}^{ \pm}, v^{ \pm}\right)
\end{array}\right)
$$

with the boundary conditions

$$
\left(\mathbf{r}^{ \pm}, s^{ \pm}\right)(0 ; \varepsilon, \rho)=\left(\mathbf{r}^{ \pm}, s^{ \pm}\right)( \pm \infty ; \varepsilon, \rho)=\mathbf{0} .
$$

$\mathbf{T}^{ \pm}\left(\mathbf{r}^{ \pm}, s^{ \pm}\right)$are differential operators from $\stackrel{\circ}{X}_{\varepsilon}\left(\mathbf{R}_{ \pm}\right)$into $Y\left(\mathbf{R}_{ \pm}\right)$, where

$$
\begin{gathered}
\stackrel{\circ}{X}_{\varepsilon}\left(\mathbf{R}_{ \pm}\right)=\stackrel{\circ}{X}_{\sigma, \varepsilon}^{2}\left(\mathbf{R}_{ \pm}\right) \times \stackrel{\circ}{X}_{\sigma, \varepsilon}^{2}\left(\mathbf{R}_{ \pm}\right) \times \stackrel{\circ}{X}_{\sigma, 1}^{2}\left(\mathbf{R}_{ \pm}\right), \\
Y\left(\mathbf{R}_{ \pm}\right)=X_{\sigma, 1}^{0}\left(\mathbf{R}_{ \pm}\right) \times X_{\sigma, 1}^{0}\left(\mathbf{R}_{ \pm}\right) \times X_{\sigma, 1}^{0}\left(\mathbf{R}_{ \pm}\right)
\end{gathered}
$$

Here $\sigma$ is an arbitrarily fixed constant satisfying $0<\sigma<\sigma_{0} . \mathbf{T}^{ \pm}\left(\mathbf{r}^{ \pm}, s^{ \pm} ; \varepsilon, \rho\right)$ have the same properties as in [I, Lemma 3.3]. Thus, we can apply the implicit function theorem to

$$
\mathbf{T}^{ \pm}\left(\mathbf{r}^{ \pm}, s^{ \pm} ; \varepsilon, \rho\right)=\mathbf{0}
$$

and we have the following lemma:

LEMMA 2.6. There are $\varepsilon_{0}>0, c_{0}>0$ such that for any $\varepsilon \in\left(0, \varepsilon_{0}\right)$ and $\rho \in \Sigma_{c_{0}}$, there exist $\left(\mathbf{r}^{ \pm}, s^{ \pm}\right)(\varepsilon, \rho) \in \dot{X}_{\varepsilon}\left(\mathbf{R}_{ \pm}\right)$satisfying $(2.10)_{ \pm} . \quad$ Moreover $\left(\mathbf{r}^{ \pm}, s^{ \pm}\right)(\varepsilon, \rho)$, $\partial\left(\mathbf{r}^{ \pm}, s^{ \pm}\right) / \partial \theta(\varepsilon, \rho), \partial\left(\mathbf{r}^{ \pm}, s^{ \pm}\right) / \partial \omega(\varepsilon, \rho)$ and $\partial\left(\mathbf{r}^{ \pm}, s^{ \pm}\right) / \partial \nu(\varepsilon, \rho)$ are uniformly continuous with respect to $(\varepsilon, \rho) \in\left(0, \varepsilon_{0}\right) \times \Sigma_{c_{0}}$ in the $\stackrel{\circ}{X}_{\varepsilon}\left(\mathbf{R}_{ \pm}\right)$-topology and satisfy

$$
\left\{\begin{array}{l}
\left\|\left(\mathbf{r}^{ \pm}, s^{ \pm}\right)(\varepsilon, \rho)\right\|_{\dot{X}_{\varepsilon}\left(\mathbf{R}_{ \pm}\right)}=o(1) \\
\left\|\partial\left(\mathbf{r}^{ \pm}, s^{ \pm}\right) / \partial \theta(\varepsilon, \rho)\right\|_{\dot{X}_{\varepsilon}\left(\mathbf{R}_{ \pm}\right)}=o(1) \\
\left\|\partial\left(\mathbf{r}^{ \pm}, s^{ \pm}\right) / \partial \omega(\varepsilon, \rho)\right\|_{\dot{X}_{\varepsilon}\left(\mathbf{R}_{ \pm}\right)}=o(1) \\
\left\|\partial\left(\mathbf{r}^{ \pm}, s^{ \pm}\right) / \partial \nu(\varepsilon, \rho)\right\|_{\dot{X}_{\varepsilon}\left(\mathbf{R}_{ \pm}\right)}=o(1)
\end{array}\right.
$$

as $\varepsilon \downarrow 0$ uniformly in $\rho \in \Sigma_{c_{0}}$.

2.4. Travelling Wave Solutions. We construct a solution of $(2.1),(2.2)$ and (2.3) in the whole interval $\mathbf{R}$, matching $\left(\mathbf{r}^{-}, s^{-}\right)(z ; \tau ; \varepsilon, \theta, \omega, \nu)$ and $\left(\mathbf{r}^{+}, s^{+}\right)(z ; \tau ; \varepsilon, \theta, \omega, \nu)$ at $z=0$ in the $C^{1}$-sense. For this purpose, we define three functions $\Phi, \Psi$ and $\Pi$ by

$$
\left\{\begin{array}{l}
\Phi(\tau ; \varepsilon ; \theta, \omega, \nu) \equiv \varepsilon \frac{d}{d z} u_{1}^{-}(0 ; \tau ; \varepsilon, \theta, \omega, \nu)-\varepsilon \frac{d}{d z} u_{1}^{+}(0 ; \tau ; \varepsilon, \theta, \omega, \nu) \\
\Psi(\tau ; \varepsilon ; \theta, \omega, \nu) \equiv \varepsilon \frac{d}{d z} u_{2}^{-}(0 ; \tau ; \varepsilon, \theta, \omega, \nu)-\varepsilon \frac{d}{d z} u_{2}^{+}(0 ; \tau ; \varepsilon, \theta, \omega, \nu) \\
\Pi(\tau ; \varepsilon ; \theta, \omega, \nu) \equiv \frac{d}{d z} v^{-}(0 ; \tau ; \varepsilon, \theta, \omega, \nu)-\frac{d}{d z} v^{+}(0 ; \tau ; \varepsilon, \theta, \omega, \nu)
\end{array}\right.
$$

and determine $\theta, \omega$ and $\nu$ as functions of $\tau$ and $\varepsilon$ such that

$$
(\Phi, \Psi, \Pi)(\tau ; \varepsilon ; \theta, \omega, \nu)=0 .
$$


Setting $E$ as $E=\left\{(\varepsilon, \theta, \omega, \nu) \mid \varepsilon \in\left(0, \varepsilon_{0}\right),(\theta, \omega, \nu) \in \Sigma_{c_{0}}\right\}$, we know form Lemma 2.6 that $(\Phi, \Psi, \Pi)(\tau ; \varepsilon ; \theta, \omega, \nu)$ is uniformly continuous in $E$. Therefore, $\Phi, \Psi$ and $\Pi$ can be continuously extended so as to be defined for $\bar{E}$. Setting $\varepsilon=0$ in (2.11), we put

$$
\left(\Phi_{0}, \Psi_{0}, \Pi_{0}\right)(\tau ; \theta, \omega, \nu)=(\Phi, \Psi, \Pi)(\tau ; 0 ; \theta, \omega, \nu)
$$

Then we easily find that

$$
\left\{\begin{array}{l}
\Phi_{0}(\tau ; \theta, \omega, \nu)=\bar{u}_{1, \xi}^{-}(0 ; \tau ; \theta, \omega, \nu)-\bar{u}_{1, \xi}^{+}(0 ; \tau ; \theta, \omega, \nu) \\
\Psi_{0}(\tau ; \theta, \omega, \nu)=\bar{u}_{2, \xi}^{-}(0 ; \tau ; \theta, \omega, \nu)-\bar{u}_{2, \xi}^{+}(0 ; \tau ; \theta, \omega, \nu) \\
\Pi_{0}(\tau ; \theta, \omega, \nu)=V_{z}^{-}(0 ; \theta, \omega)-V_{z}^{+}(0 ; \theta, \omega)
\end{array}\right.
$$

Lemmas 2.2 and 2.5 show that the relation $\left(\Phi_{0}, \Psi_{0}, \Pi_{0}\right)(\tau ; \theta, \omega, \nu)=0$ is equivalent to the conditions

$$
\omega=\omega_{O}(\theta), \quad \theta=\theta_{I}(\tau ; \omega)=\bar{\Theta}(\omega) / \tau
$$

and $\nu=\bar{u}_{2}(0 ; \omega)$. By Lemma 2.2, we can rewrite $\omega=\omega_{O}(\theta)$ inversely as $\theta=\theta_{O}(\omega)$ for $\omega \in\left(p_{-}, p_{+}\right)$. We know the shape of the curve $\theta=\theta_{O}(\omega)$ well. Let us examine that of the curve $\theta=\theta_{I}(\tau ; \omega)=\bar{\Theta}(\omega) / \tau$. By short computation, we find that the case (H4-a) (resp. (H4-b)) implies $\alpha_{23}>\alpha_{13}$ (resp. $\alpha_{13}>\alpha_{23}$ ). In these two cases, we know that $\bar{\Theta}\left(q_{-}\right)>0$ and $\bar{\Theta}\left(q_{+}\right)<0$ because the state $\left(0,1-\alpha_{23} \omega\right)$ is dominant when $\omega=q_{-}$and the state $\left(1-\alpha_{13} \omega, 0\right)$ is dominant when $\omega=q_{+}$for the subsystem $\varepsilon \tau \mathbf{u}_{t}=\varepsilon^{2} D \mathbf{u}_{x x}+\mathbf{f}(\mathbf{u}, \omega)$. Furthermore we easily find that there exits only one $\omega_{0} \in I\left(q_{-} \sim q_{+}\right)$such that $\bar{\Theta}\left(\omega_{0}\right)=0$ by virtue of Lemma 2.4 (see Fig.2.2, Fig.2.3, Fig.2.4 and Fig.2.5).

First, let us consider the case (H4-a). If $\omega_{0} \in\left(q_{-}, q_{+}\right) \backslash I\left(p_{-} \sim p_{+}\right)$, there exists only one intersection of the curves $\theta=\theta_{O}(\omega)$ and $\theta=\theta_{I}(\tau ; \omega)$ at which these intersect transversally for small or large $\tau>0$ (see Fig.2.2). On the other hand, if $\omega_{0} \in I\left(p_{-} \sim\right.$ $\left.p_{+}\right)$, there exists one transversal intersection for large $\tau>0$ and three transversal intersections for small $\tau>0$ (see Fig.2.3). In the case (H4-b), there exists one transversal intersection for small or large $\tau>0$ (see Fig.2.4 and Fig.2.5). Finally, let us consider the case (H4-c) in which there are several possibility. When $\alpha_{13}>\alpha_{23}$, we know that $\min \left\{q_{-}, q_{+}\right\}=q_{-}$and $\bar{\Theta}\left(q_{-}\right)>0$, which implies that there exists one transversal intersection for small or large $\tau>0$ (see Fig.2.6). When $\alpha_{13}<\alpha_{23}$, we find that $\min \left\{q_{-}, q_{+}\right\}=q_{+}$and $\bar{\Theta}\left(q_{+}\right)<0$. Then if there exists $\omega_{0} \in I\left(p_{-} \sim p_{+}\right)$ such that $\bar{\Theta}\left(\omega_{0}\right)=0$, there exists one transversal intersection for large $\tau>0$ and three transversal intersections for small $\tau>0$ (see Fig.2.7). For other cases, there exists one transversal intersection for small or large $\tau>0$. On the above discussion, we used the following property: $\theta=\theta_{I}(\tau ; \omega)$ behaves like a monotone function of $\omega$ in a neighborhood of intersections when $\tau>0$ is small or large. But we can not say the number of intersections exactly for intermediate $\tau>0$.

We fix $\tau>0$ small or large and let $\left(\theta^{*}(\tau), \omega^{*}(\tau)\right)$ be an arbitrary intersection of the curves $\theta=\theta_{O}(\omega)$ and $\theta=\theta_{I}(\tau ; \omega)$ at which these intersect transversally. Moreover 


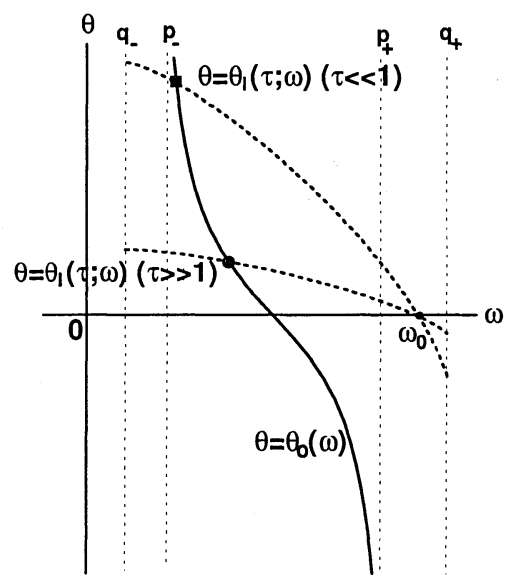

FIG. 2.2. The graphs of $\theta=\theta_{O}(\omega)$ and $\theta=\theta_{I}(\tau ; \omega)$ for small or large $\tau>0$. $\omega_{0} \in$ $\left(q_{-}, q_{+}\right) \backslash I\left(p_{-} \sim p_{+}\right)$in (H4-a).

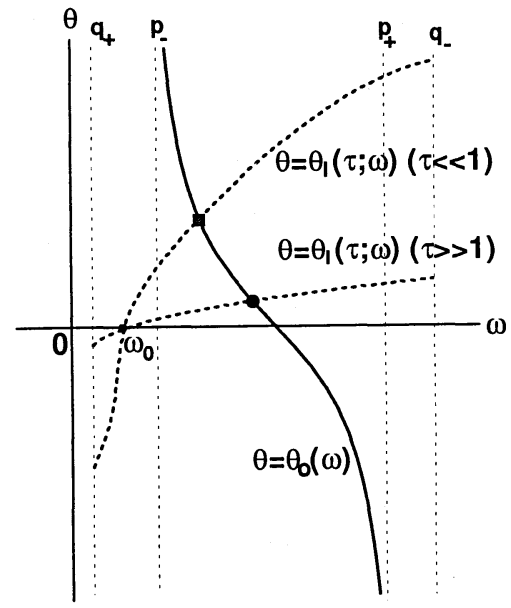

FIG. 2.4. The graphs of $\theta=\theta_{O}(\omega)$ and $\theta=\theta_{I}(\tau ; \omega)$ for small or large $\tau>0$. $\omega_{0} \in$ $\left(q_{-}, q_{+}\right) \backslash I\left(p_{-} \sim p_{+}\right)$in $\left(H_{4}-b\right)$.

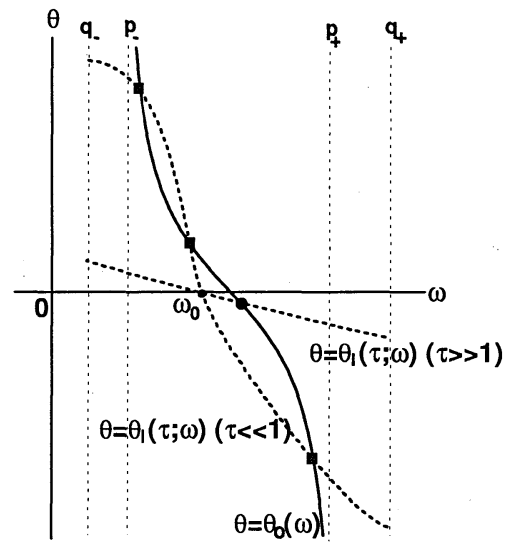

FIG. 2.3. The graphs of $\theta=\theta_{O}(\omega)$ and $\theta=\theta_{I}(\tau ; \omega)$ for small or large $\tau>0$. $\omega_{0} \in I\left(p_{-} \sim p_{+}\right)$in $\left(H_{4}-a\right)$.

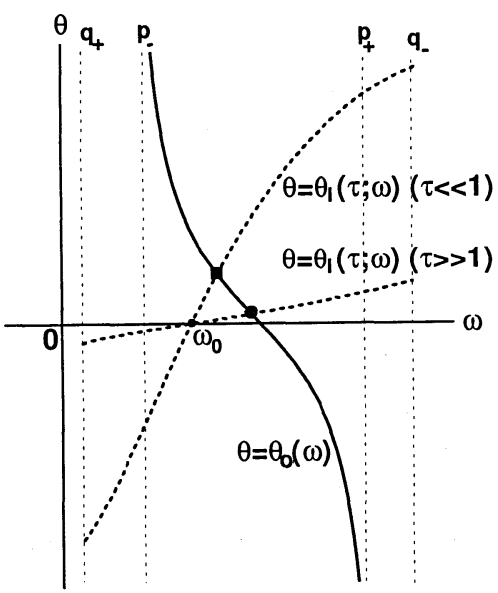

FIG. 2.5. The graphs of $\theta=\theta_{O}(\omega)$ and $\theta=\theta_{I}(\tau ; \omega)$ for small or large $\tau>0$. $\omega_{0} \in I\left(p_{-} \sim p_{+}\right)$in $\left(H_{4}-b\right)$.

we define $\nu^{*}(\tau)=\bar{u}_{2}\left(0 ; \omega^{*}(\tau)\right)$. Then from Lemmas 2.1, 2.2, 2.3 and 2.5, we find that

$$
\begin{gathered}
\Phi_{0}\left(\tau ; \theta^{*}(\tau), \omega^{*}(\tau), \nu^{*}(\tau)\right)=0, \Psi_{0}\left(\tau ; \theta^{*}(\tau), \omega^{*}(\tau), \nu^{*}(\tau)\right)=0, \\
\Pi_{0}\left(\tau ; \theta^{*}(\tau), \omega^{*}(\tau), \nu^{*}(\tau)\right)=0,
\end{gathered}
$$$$
\frac{\partial}{\partial \theta} \Pi_{0}\left(\tau ; \theta^{*}(\tau), \omega^{*}(\tau), \nu^{*}(\tau)\right)>0, \frac{\partial}{\partial \omega} \Pi_{0}\left(\tau ; \theta^{*}(\tau), \omega^{*}(\tau), \nu^{*}(\tau)\right)>0
$$$$
\frac{\partial}{\partial \nu} \Pi_{0}\left(\tau ; \theta^{*}(\tau), \omega^{*}(\tau), \nu^{*}(\tau)\right)=0
$$$$
\frac{\partial}{\partial \theta} \Phi_{0}\left(\tau ; \theta^{*}(\tau), \omega^{*}(\tau), \nu^{*}(\tau)\right) p_{1}^{*}(0)+d \frac{\partial}{\partial \theta} \Psi_{0}\left(\tau ; \theta^{*}(\tau), \omega^{*}(\tau), \nu^{*}(\tau)\right) p_{2}^{*}(0)
$$$$
=\tau \int_{-\infty}^{\infty}\left\{\bar{u}_{1, \xi}(\xi) p_{1}^{*}(\xi)+\bar{u}_{2, \xi}(\xi) p_{2}^{*}(\xi)\right\} d \xi,
$$$$
\frac{\partial}{\partial \omega} \Phi_{0}\left(\tau ; \theta^{*}(\tau), \omega^{*}(\tau), \nu^{*}(\tau)\right) p_{1}^{*}(0)+d \frac{\partial}{\partial \omega} \Psi_{0}\left(\tau ; \theta^{*}(\tau), \omega^{*}(\tau), \nu^{*}(\tau)\right) p_{2}^{*}(0)
$$$$
=\int_{-\infty}^{\infty}\left\{a \alpha_{13} \bar{u}_{1}(\xi) p_{1}^{*}(\xi)+b \alpha_{23} \bar{u}_{2}(\xi) p_{2}^{*}(\xi)\right\} d \xi,
$$

$\frac{\partial}{\partial \nu} \Phi_{0}\left(\tau ; \theta^{*}(\tau), \omega^{*}(\tau), \nu^{*}(\tau)\right) p_{1}^{*}(0)+d \frac{\partial}{\partial \nu} \Psi_{0}\left(\tau ; \theta^{*}(\tau), \omega^{*}(\tau), \nu^{*}(\tau)\right) p_{2}^{*}(0)=0$,

$\frac{\partial}{\partial \omega} \theta_{I}\left(\tau ; \omega^{*}(\tau)\right)-\frac{\partial}{\partial \omega} \theta_{O}\left(\omega^{*}(\tau)\right) \neq 0$. 


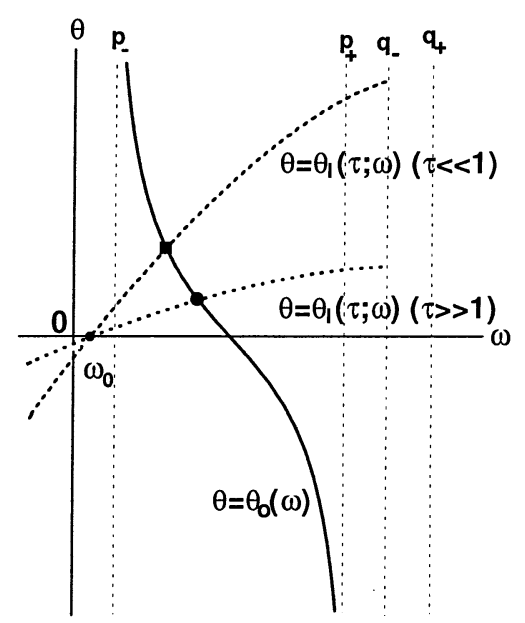

FIG. 2.6. The graphs of $\theta=\theta_{O}(\omega)$ and $\theta=\theta_{I}(\tau ; \omega)$ for small or large $\tau>0 . \alpha_{13}>$ $\alpha_{23}$ and $\omega_{0} \notin I\left(p_{-} \sim p_{+}\right)$in (H4-c).

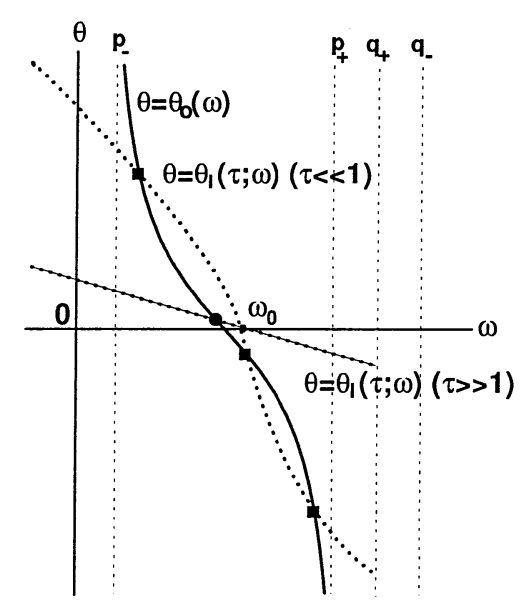

FIG. 2.7. The graphs of $\theta=\theta_{O}(\omega)$ and $\theta=\theta_{I}(\tau ; \omega)$ for small or large $\tau>0$. $\alpha_{13}<\alpha_{23}$ and $\omega_{0} \in I\left(p_{-} \sim p_{+}\right)$in (H4c).

These imply that

$$
\begin{aligned}
\operatorname{det}\left\{\frac{\partial\left(\Phi_{0}, \Psi_{0}, \Omega_{0}\right)}{\partial(\theta, \omega, \nu)}\left(\tau ; \theta^{*}(\tau), \omega^{*}(\tau), \nu^{*}(\tau)\right)\right\}= \\
-\tau \frac{\partial}{\partial \nu} \Psi_{0}\left(\tau ; \theta^{*}(\tau), \omega^{*}(\tau), \nu^{*}(\tau)\right) \cdot \frac{\partial}{\partial \theta} \Pi_{0}\left(\tau ; \theta^{*}(\tau), \omega^{*}(\tau), \nu^{*}(\tau)\right) \int_{-\infty}^{\infty}\left\{\bar{u}_{1, \xi}(\xi) p_{1}^{*}(\xi)\right. \\
\left.\quad+\bar{u}_{2, \xi}(\xi) p_{2}^{*}(\xi)\right\} d \xi\left\{\frac{\partial}{\partial \omega} \theta_{I}\left(\tau ; \omega^{*}(\tau)\right)-\frac{\partial}{\partial \omega} \theta_{O}\left(\omega^{*}(\tau)\right)\right\} / p_{1}^{*}(0) \neq 0 .
\end{aligned}
$$

Here we used the relation $\frac{\partial}{\partial \nu} \Psi_{0}\left(\tau ; \theta^{*}(\tau), \omega^{*}(\tau), \nu^{*}(\tau)\right) \neq 0$. Therefore we can apply the implicit function theorem to $(2.12)$ and we have the next lemma.

LEMMA 2.7. Let $\left(\theta^{*}(\tau), \omega^{*}(\tau)\right)$ be an arbitrary intersection of the curves $\theta=\theta_{O}(\omega)$ and $\theta=\theta_{I}(\tau ; \omega)$ at which these intersect transversally, and define $\nu^{*}(\tau)=$ $\bar{u}_{2}\left(0 ; \omega^{*}(\tau)\right)$. Then there is $\varepsilon_{0}>0$ such that for any $\varepsilon \in\left[0, \varepsilon_{0}\right)$, there exist $\theta(\tau ; \varepsilon)$, $\omega(\tau ; \varepsilon)$ and $\nu(\tau ; \varepsilon)$ satisfying

$$
(\Phi, \Psi, \Pi)(\tau ; \varepsilon ; \theta(\tau ; \varepsilon), \omega(\tau ; \varepsilon), \nu(\tau ; \varepsilon))=0
$$

and

$$
\lim _{\varepsilon \downarrow 0} \theta(\tau ; \varepsilon)=\theta^{*}(\tau), \lim _{\varepsilon \downarrow 0} \omega(\tau ; \varepsilon)=\omega^{*}(\tau), \lim _{\varepsilon \downarrow 0} \nu(\tau ; \varepsilon)=\nu^{*}(\tau)
$$

We obtain our results.

ThEOREM 2.8. Suppose that (H1),(H2),(H3) and (H4) hold. When $\omega_{0} \in$ $I\left(p_{-} \sim p_{+}\right)$in (H4-a) or $\alpha_{13}<\alpha_{23}$ and $\omega_{0} \in I\left(p_{-} \sim p_{+}\right)$in (H4-c), where $\omega_{0}$ satisfies $\bar{\Theta}\left(\omega_{0}\right)=0$, there is $\varepsilon_{0}>0$ such that for any $\varepsilon \in\left(0, \varepsilon_{0}\right)$, there exists $\theta=\theta_{i}(\tau ; \varepsilon)\left(\theta_{1}<\right.$ $\left.\theta_{2}<\theta_{3}, \theta_{1} \theta_{3}<0\right)$ such that the problem (2.1),(2.2) and (2.3) has three solutions 
$\left(\mathbf{u}_{i}, v_{i}\right)(z ; \tau ; \varepsilon)$ with velocity $\theta_{i}(\tau ; \varepsilon) \rightarrow \theta_{i}^{*}(\tau)$ as $\varepsilon \downarrow 0$ for small $\tau>0(i=1,2,3)$, and only one solution for large $\tau>0$. For other cases in (H4), the problem (2.1), (2.2) and (2.3) has only one solution for small or large $\tau>0$.

3. Stability. By the travelling coordinate system $(t, z)=(t, x+\theta t),(1.3)$ takes the form

$$
\left\{\begin{array}{l}
\varepsilon \tau \mathbf{u}_{t}=\varepsilon^{2} D \mathbf{u}_{z z}-\varepsilon \tau \theta \mathbf{u}_{z}+\mathbf{f}(\mathbf{u}, v) \\
v_{t}=v_{z z}-\theta v_{z}+g(\mathbf{u}, v)
\end{array},(t, z) \in \mathbf{R}_{+} \times \mathbf{R} .\right.
$$

Then, the travelling front solution $(\mathbf{u}, v)(z ; \varepsilon)$ obtained in the previous section is a stationary solution of (3.1). Here we will study the stability of this stationary solution. For this purpose, it is enough to examine the distribution of isolated eigenvalues of the following linearized eigenvalue problem of $(3.1)$ around $(\mathbf{u}, v)(z ; \varepsilon)$ :

$$
\left\{\begin{array}{l}
\varepsilon \tau \lambda \mathbf{w}=\varepsilon^{2} D \mathbf{w}_{z z}-\varepsilon \tau \theta \mathbf{w}_{z}+\mathbf{f}_{\mathbf{u}}^{\varepsilon}(z) \mathbf{w}+\mathbf{f}_{v}^{\varepsilon}(z) y \\
\lambda y=y_{z z}-\theta y_{z}+g_{\mathbf{u}}^{\varepsilon}(z) \cdot \mathbf{w}+g_{v}^{\varepsilon}(z) y
\end{array}, z \in \mathbf{R}\right.
$$

and $(\mathbf{w}, y)(z ; \varepsilon ; \lambda) \in(B C(\mathbf{R}))^{3}$, where $\mathbf{w}={ }^{t}\left(w_{1}, w_{2}\right), \mathbf{u}={ }^{t}\left(u_{1}, u_{2}\right), \mathbf{f}={ }^{t}\left(f_{1}, f_{2}\right)$,

$$
\mathbf{f}_{\mathbf{u}}^{\varepsilon}(z)=\left(\begin{array}{ll}
f_{1 u_{1}}(\mathbf{u}(z ; \varepsilon), v(z ; \varepsilon)) & f_{1 u_{2}}(\mathbf{u}(z ; \varepsilon), v(z ; \varepsilon)) \\
f_{2 u_{1}}(\mathbf{u}(z ; \varepsilon), v(z ; \varepsilon)) & f_{2 u_{2}}(\mathbf{u}(z ; \varepsilon), v(z ; \varepsilon))
\end{array}\right)
$$

and the other functions $\mathbf{f}_{v}^{\varepsilon}(z), g_{\mathbf{u}}^{\varepsilon}(z)$ and $g_{v}^{\varepsilon}(z)$ are similarly defined. $\cdot$ means the usual inner product in $\mathbf{R}^{2}$.

We define the operators $L^{\varepsilon}$ and $L^{\varepsilon, *}$ by

$$
L^{\varepsilon} \mathbf{w}=\varepsilon^{2} D \mathbf{w}_{z z}-\varepsilon \tau \theta \mathbf{w}_{z}+\mathbf{f}_{\mathbf{u}}^{\varepsilon}(z) \mathbf{w} \quad \text { and } \quad L^{\varepsilon, *} \mathbf{w}=\varepsilon^{2} D \mathbf{w}_{z z}+\varepsilon \tau \theta \mathbf{w}_{z}+{ }^{t} \mathbf{f}_{\mathbf{u}}^{\varepsilon}(z) \mathbf{w}
$$

respectively. Let $\left\{\zeta_{n}^{\varepsilon}, \phi_{n}^{\varepsilon}\right\}$ (resp. $\left.\left\{\zeta_{n}^{\varepsilon, *}, \phi_{n}^{\varepsilon, *}\right\}\right)$ be eigenpairs of the eigenvalue problem

$$
\left\{\begin{array} { l } 
{ L ^ { \varepsilon } \phi = \zeta \phi , z \in \mathbf { R } } \\
{ \phi , \phi _ { z } , \phi _ { z z } \in B C ( \mathbf { R } ) , }
\end{array} \quad \left(\operatorname{resp} .\left\{\begin{array}{l}
L^{\varepsilon, *} \phi^{*}=\zeta^{*} \phi^{*}, z \in \mathbf{R} \\
\phi^{*}, \phi_{z}^{*}, \phi_{z z}^{*} \in B C(\mathbf{R}),
\end{array}\right)\right.\right.
$$

where $\left\{\phi_{n}^{\varepsilon}\right\}$ and $\left\{\phi_{n}^{\varepsilon, *}\right\}$ are normalized as $\left\|\phi_{n}^{\varepsilon}\right\|_{L^{2}(\mathbf{R})}=1$ and $\left\langle\phi_{n}^{\varepsilon}, \phi_{n}^{\varepsilon, *}>_{L^{2}(\mathbf{R})}=\right.$ $1(n=0,1,2, \ldots)$. Assume that $\operatorname{Re} \zeta_{n}^{\varepsilon} \leq \operatorname{Re} \zeta_{0}^{\varepsilon}(n \geq 1)$. Then we easily find that

$$
\zeta_{n}^{\varepsilon, *}=\overline{\zeta_{n}^{\varepsilon}} \quad(n \geq 0) \text {. }
$$

LEMMA 3.1. $\quad\left\{\zeta_{n}^{\varepsilon}\right\}$ satisfy the following properties:

(i) $\operatorname{Re} \zeta_{0}^{\varepsilon}=\varepsilon L(\varepsilon)$ and $\operatorname{Im} \zeta_{0}^{\varepsilon}=o(\varepsilon)$ as $\varepsilon \rightarrow 0$, where

$$
\begin{gathered}
L(0)=-\left\{\theta^{*}(\tau)\left(\omega^{*}(\tau)-p_{-}\right)\right. \\
\left.-\int_{-\infty}^{0} g\left(\mathbf{U}^{-}\left(z ; \theta^{*}(\tau), \omega^{*}(\tau)\right), V^{-}\left(z ; \theta^{*}(\tau), \omega^{*}(\tau)\right)\right) d z\right\} \frac{\partial \bar{\Theta}}{\partial \omega}\left(\omega^{*}(\tau)\right) .
\end{gathered}
$$

(ii) There are $\varepsilon_{0}>0$ and $d_{0}>0$ such that $\operatorname{Re} \zeta_{n}^{\varepsilon}<-d_{0}$ for any $\varepsilon \in\left(0, \varepsilon_{0}\right)$ and $n \geq 1$. 
We can prove this in a way similar to that of Lemma 3.2 in [NMIF], so we omit it.

REMARK 3.2. The sign of $L(0)$ is not definite (cf. [NMIF, Lemma 3.2]). In fact,

$$
\operatorname{sgn}\{L(0)\}=-\operatorname{sgn}\left\{\frac{\partial \bar{\Theta}}{\partial \omega}\left(\omega^{*}(\tau)\right)\right\}
$$

where $\operatorname{sgn}\{a\}=1$ for $a>0,=-1$ for $a<0,=0$ for $a=0$.

Suppose that there is an isolated eigenvalue $\lambda \in \mathbf{C}_{d_{0}} \equiv\left\{\lambda \in \mathbf{C} \mid \operatorname{Re} \lambda>-d_{0}\right\}$ $\left(d_{0}>0\right)$ of $(3.2)$ with the corresponding eigenfunction $(\mathbf{w}, y)(z ; \varepsilon ; \lambda) \in(B C(\mathbf{R}))^{3}$. Then $(\mathbf{w}, y)$ must decay with the exponential order as $|z| \rightarrow \infty$ by (H2),(H3) and (H4). This guarantees us that we will be able to work in much more convenient Hilbert spase $H^{1}(\mathbf{R})$ for isolated eigenvalues. Applying the SLEP method which is developed by Nishiura and Fujii [NF] (see also [T]), we will calculate the distribution of isolated eigenvalues of (3.2). Let $\Sigma^{\varepsilon}$ be the set of all eigenvalues of (3.2) for $\varepsilon \in\left(0, \varepsilon_{0}\right)$. Lemma 2.1 in $[\mathrm{NF}]$ says that $\zeta_{0}^{\varepsilon} / \varepsilon \tau \notin \Sigma^{\varepsilon}$ for $\varepsilon \in\left(0, \varepsilon_{0}\right)$. Then we can solve the first equation of (3.2) with respect to $\mathbf{w}$ for $\lambda \in \mathbf{C}_{d_{0}} \cap \Sigma^{\varepsilon}$ :

$$
\mathbf{w}=\left(L^{\varepsilon}-\varepsilon \tau \lambda\right)^{-1}\left(-\mathbf{f}_{v}^{\varepsilon} y\right) .
$$

Let $P^{\varepsilon}$ be the projection operator onto the eigenspace $\left\{\phi_{0}^{\varepsilon}\right\}$ :

$$
P^{\varepsilon}(\cdot)=<\cdot, \phi_{0}^{\varepsilon, *}>\phi_{0}^{\varepsilon},
$$

and decompose $\left(L^{\varepsilon}-\varepsilon \tau \lambda\right)^{-1}$ into two parts

$$
\left(L^{\varepsilon}-\varepsilon \tau \lambda\right)^{-1}(\cdot)=\frac{1}{\zeta_{0}^{\varepsilon}-\varepsilon \tau \lambda} P^{\varepsilon}(\cdot)+\left(L^{\varepsilon}-\varepsilon \tau \lambda\right)^{\dagger}(\cdot) .
$$

Then we know that there exists a positive constant $M$ satisfying

$$
\left\|\left(L^{\varepsilon}-\varepsilon \tau \lambda\right)^{\dagger}\right\|_{L^{2}(\mathbf{R}) \rightarrow L^{2}(\mathbf{R})} \leq M
$$

for any $\varepsilon \in\left(0, \varepsilon_{0}\right), \lambda \in \mathbf{C}_{d_{0}} \cap \Sigma^{\varepsilon}$. w is represented explicitly as

$$
\mathbf{w}=-\frac{\left\langle\mathbf{f}_{v}^{\varepsilon} y, \phi_{0}^{\varepsilon, *}>\right.}{\zeta_{0}^{\varepsilon}-\varepsilon \tau \lambda} \phi_{0}^{\varepsilon}-\left(L^{\varepsilon}-\varepsilon \tau \lambda\right)^{\dagger}\left(\mathbf{f}_{v}^{\varepsilon} y\right) .
$$

Substituting (3.3) into the second equation of (3.2), we have the eigenvalue problem with respect to $y \in H^{1}(\mathbf{R})$ :

$$
y_{z z}-\theta y_{z}-\frac{\left\langle\mathbf{f}_{v}^{\varepsilon} y, \phi_{0}^{\varepsilon, *}\right\rangle}{\zeta_{0}^{\varepsilon}-\varepsilon \tau \lambda} g_{\mathbf{u}}^{\varepsilon} \cdot \phi_{0}^{\varepsilon}-g_{\mathbf{u}}^{\varepsilon}\left(L^{\varepsilon}-\varepsilon \tau \lambda\right)^{\dagger}\left(\mathbf{f}_{v}^{\varepsilon} y\right)+g_{v}^{\varepsilon} y=\lambda y \quad, z \in \mathbf{R} .
$$

The core of the SLEP method consists of the following two key lemmas, which characterize the asymptotic behaviours of the second and the third terms of the left-hand side of (3.4).

Lemma 3.3. (The first key lemma.) ([T, Theorem 2]) For any $y \in L^{2}(\mathbf{R})$ and any $s \in(0,1 / 2)$,

$$
\left(L^{\varepsilon}-\varepsilon \tau \lambda\right)^{\dagger}\left(\mathbf{f}_{v}^{\varepsilon} y\right) \longrightarrow\left(\mathbf{f}_{\mathbf{u}}^{0}\right)^{-1}\left(\mathbf{f}_{v}^{0} y\right) \text { as } \varepsilon \rightarrow 0
$$


strongly in $\left(H^{s}\right)^{\sharp}(\mathbf{R})$-sense and uniformly in $\lambda \in \mathbf{C}_{d_{0}}$, where

$$
\mathbf{f}_{\mathbf{u}}^{0}=\left(\begin{array}{ll}
f_{1 u_{1}}(*) & f_{1 u_{2}}(*) \\
f_{2 u_{1}}(*) & f_{2 u_{2}}(*)
\end{array}\right), \mathbf{f}_{v}^{0}={ }^{t}\left(f_{1 v}(*), f_{2 v}(*)\right), *=\left(\mathbf{U}^{ \pm}, V^{ \pm}\right)(z), z \in \mathbf{R}_{ \pm}
$$

Moreover, the above convergence is uniform on a bounded set in $\mathbf{C}_{d_{0}} \times L^{2}(\mathbf{R})$.

LEMma 3.4.(The second key lemma.) ([NF, Lemma 2.3]) As $\varepsilon \rightarrow 0$,

(i) $\quad \frac{1}{\sqrt{\varepsilon}} \mathbf{f}_{v}^{\varepsilon} \cdot \phi_{0}^{\varepsilon, *} \longrightarrow \frac{\partial \bar{\Theta}}{\partial \omega}\left(\omega^{*}(\tau)\right) \delta_{0}$,

(ii) $\quad \frac{1}{\sqrt{\varepsilon}} g_{\mathbf{u}}^{\varepsilon} \cdot \phi_{0}^{\varepsilon} \longrightarrow\left[g\left(0,1-\alpha_{23} \omega^{*}(\tau), \omega^{*}(\tau)\right)-g\left(1-\alpha_{13} \omega^{*}(\tau), 0, \omega^{*}(\tau)\right)\right] \delta_{0}$

in $\left(H^{1}\right)^{\sharp}(\mathbf{R})$-sense uniformly for $\lambda \in \mathbf{C}_{d_{0}}$, where $\delta_{0}=\delta(z)$ is a Dirac's $\delta$-function at 0 .

Then we can take the limit $\varepsilon \rightarrow 0$ of $(3.4)$ in $\left(H^{1}\right)^{\sharp}(\mathbf{R})$, and get the singular limit eigenvalue problem (SLEP):

$$
y_{z z}-\theta^{*}(\tau) y_{z}-\frac{c^{*}<y, \delta_{0}>}{L(0)-\tau \lambda} \delta_{0}-g_{\mathbf{u}}^{0}\left(\mathbf{f}_{\mathbf{u}}^{0}\right)^{-1} \mathbf{f}_{v}^{0} y+g_{v}^{0} y=\lambda y, y \in H^{1}(\mathbf{R})
$$

where

$$
c^{*}=\frac{\partial \bar{\Theta}}{\partial \omega}\left(\omega^{*}(\tau)\right)\left[g\left(0,1-\alpha_{23} \omega^{*}(\tau), \omega^{*}(\tau)\right)-g\left(1-\alpha_{13} \omega^{*}(\tau), 0, \omega^{*}(\tau)\right)\right],
$$

which is identical to the relation

$$
y_{z z}-\theta^{*}(\tau) y_{z}-\frac{c^{*}<y, \delta_{0}>}{L(0)-\tau \lambda} \delta_{0}+\operatorname{det}^{*} y=\lambda y, y \in H^{1}\left(\mathbf{R}_{ \pm}\right)
$$

where

$$
\operatorname{det}^{*}=g_{v}^{0}-g_{\mathbf{u}}^{0}\left(\mathbf{f}_{\mathbf{u}}^{0}\right)^{-1} \mathbf{f}_{v}^{0}<0,
$$

and $g_{\mathbf{u}}^{0}$ and $g_{v}^{0}$ are defined similarly to $\mathbf{f}_{\mathbf{u}}^{0}$ and $\mathbf{f}_{v}^{0}$. Without loss of generality, we can normalize the limiting eigenfunction $y$ as $\left\langle y, \delta_{0}\right\rangle=y(0)=1$. Then (3.6) is equivalent to the following equations:

$$
\left\{\begin{array}{l}
\frac{d^{2}}{d z^{2}} y^{ \pm}-\theta^{*}(\tau) \frac{d}{d z} y^{ \pm}+\operatorname{det}^{*} y^{ \pm}=\lambda y^{ \pm}, y^{ \pm} \in H^{1}\left(\mathbf{R}_{ \pm}\right) \\
y^{-}(0)=1=y^{+}(0) \\
\frac{d}{d z} y^{+}(0)-\frac{d}{d z} y^{-}(0)=\frac{c^{*}}{L(0)-\tau \lambda}
\end{array}\right.
$$

where

$$
\begin{aligned}
& \frac{c^{*}}{L(0)-\tau \lambda} \\
& =\frac{\left[g\left(0,1-\alpha_{23} \omega^{*}(\tau), \omega^{*}(\tau)\right)-g\left(1-\alpha_{13} \omega^{*}(\tau), 0, \omega^{*}(\tau)\right)\right]}{\int_{-\infty}^{0} g\left(U_{1}^{-}\left(z ; \omega^{*}(\tau)\right), U_{2}^{-}\left(z ; \omega^{*}(\tau)\right), V^{-}\left(z ; \omega^{*}(\tau)\right)\right) d z-\tau \lambda / \frac{\partial \bar{\Theta}}{\partial \omega}\left(\omega^{*}(\tau)\right)} .
\end{aligned}
$$


To get an information of the dependency of $\lambda$ on $\varepsilon>0$, we convert the SLEP equation (3.5) into the equivalent transcendental equation. Let us introduce the differential operator $T_{\lambda}^{\varepsilon, \tau, \theta}: H^{1}(\mathbf{R}) \rightarrow\left(H^{1}\right)^{\sharp}(\mathbf{R})$;

$$
T_{\lambda}^{\varepsilon, \tau, \theta} \equiv-\frac{d^{2}}{d z^{2}}+\theta \frac{d}{d z}+g_{\mathbf{u}}^{\varepsilon}\left(L^{\varepsilon}-\varepsilon \tau \lambda\right)^{\dagger}\left(\mathbf{f}_{v}^{\varepsilon} \cdot\right)-g_{v}^{\varepsilon}+\lambda
$$

LEMMA 3.5. ([NF, Lemma 3.1],[NMIF, Lemma 3.6]) There exists a positive constant $\varepsilon_{0}$ such that the differential operator $T_{\lambda}^{\varepsilon, \tau, \theta}$ has a uniformly bounded inverse $K_{\lambda}^{\varepsilon, \tau, \theta}:\left(H^{1}\right)^{\sharp}(\mathbf{R}) \rightarrow H^{1}(\mathbf{R})$ for any $\varepsilon \in\left(0, \varepsilon_{0}\right)$, which is continuous on $\varepsilon \in\left[0, \varepsilon_{0}\right)$ and analytic on $\lambda \in \mathbf{C}$ in the operator norm sense.

(3.4) is rewritten as

$$
T_{\lambda}^{\varepsilon, \tau, \theta} y=-\frac{<\mathbf{f}_{v}^{\varepsilon} y, \phi_{0}^{\varepsilon, *}>}{\zeta_{0}^{\varepsilon}-\varepsilon \tau \lambda} g_{\mathbf{u}}^{\varepsilon} \cdot \phi_{0}^{\varepsilon} .
$$

Then, applying the operator $K_{\lambda}^{\varepsilon, \tau, \theta}$ to this equation, we have

$$
y=-\frac{\left\langle\mathbf{f}_{v}^{\varepsilon} y, \phi_{0}^{\varepsilon, *} / \sqrt{\varepsilon}>\right.}{\zeta_{0}^{\varepsilon} / \varepsilon-\tau \lambda} K_{\lambda}^{\varepsilon, \tau, \theta}\left(g_{\mathbf{u}}^{\varepsilon} \cdot \phi_{0}^{\varepsilon} / \sqrt{\varepsilon}\right)
$$

which imples that $y$ is a constant multiple of $K_{\lambda}^{\varepsilon, \tau, \theta}\left(g_{\mathbf{u}}^{\varepsilon} \cdot \phi_{0}^{\varepsilon} / \sqrt{\varepsilon}\right)$, that is, for a constant $\alpha$

$$
y=\alpha K_{\lambda}^{\varepsilon, \tau, \theta}\left(g_{\mathbf{u}}^{\varepsilon} \cdot \phi_{0}^{\varepsilon} / \sqrt{\varepsilon}\right) \in H^{1}(\mathbf{R}) .
$$

Substituting (3.10) into (3.9), we see that a nontrivial solution $y$ of (3.9) exists if and only if $\lambda$ satisfies the algebraic-like equation

$$
\frac{\zeta_{0}^{\varepsilon}}{\varepsilon}-\tau \lambda=\left\langle K_{\lambda}^{\varepsilon, \tau, \theta}\left(g_{\mathbf{u}}^{\varepsilon} \cdot \frac{\phi_{0}^{\varepsilon}}{\sqrt{\varepsilon}}\right),-\mathbf{f}_{v}^{\varepsilon} \frac{\phi_{0}^{\varepsilon, *}}{\sqrt{\varepsilon}}\right\rangle .
$$

Then we put

$$
\mathcal{F}(\lambda ; \tau, \theta, \varepsilon) \equiv \frac{\zeta_{0}^{\varepsilon}}{\varepsilon}-\tau \lambda-\left\langle K_{\lambda}^{\varepsilon, \tau, \theta}\left(g_{\mathbf{u}}^{\varepsilon} \cdot \frac{\phi_{0}^{\varepsilon}}{\sqrt{\varepsilon}}\right),-\mathbf{f}_{v}^{\varepsilon} \frac{\phi_{0}^{\varepsilon, *}}{\sqrt{\varepsilon}}\right\rangle=0
$$

Lemmas 3.1, 3.4 and 3.5 guarantee us to be able to take the limit $\varepsilon \rightarrow 0$ in (3.12). Thus the limiting equation is give by

$$
\mathcal{F}_{0}\left(\lambda ; \tau, \theta^{*}(\tau)\right) \equiv \mathcal{F}\left(\lambda ; \tau, \theta^{*}(\tau), 0\right)=L(0)-\tau \lambda+c^{*}<K_{\lambda}^{0, \tau, \theta^{*}(\tau)} \delta_{0}, \delta_{0}>=0 .
$$

Applying the same method as in the proof of Lemma 3.7 in [NMIF], we can show that $\mathcal{F}_{0}\left(\lambda ; \tau, \theta^{*}(\tau)\right)=0$ has no complex roots in $\mathbf{C}_{d_{0}}$. In order to examine real roots, we put $G\left(\lambda ; \tau, \theta^{*}(\tau)\right) \equiv-c^{*}<K_{\lambda}^{0, \tau, \theta^{*}(\tau)} \delta_{0}, \delta_{0}>$. Then we have the next lemma.

LEMma 3.6. ([NMIF, Lemma 3.8]) If $L(0)=0, G\left(\lambda ; \tau, \theta^{*}(\tau)\right) \equiv 0$. Suppose that $L(0) \neq 0 . G\left(\lambda ; \tau, \theta^{*}(\tau)\right)$ is a strictly monotone and convex function of real $\lambda$ for $\lambda>-d_{0}$, and satisfies $G\left(0 ; \tau, \theta^{*}(\tau)\right)=L(0)$ and $\lim _{\lambda \rightarrow \infty} G\left(\lambda ; \tau, \theta^{*}(\tau)\right)=0$ (see Fig.3.1 and Fig.3.2). 


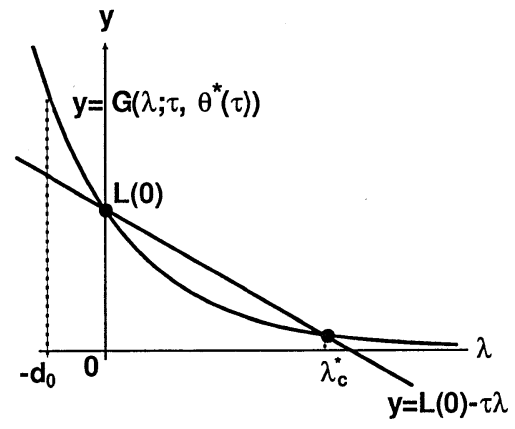

FIG. 3.1. The graphs of $y=G\left(\lambda ; \tau, \theta^{*}(\tau)\right)$ and $y=L(0)-\tau \lambda$ when $L(0)>0$

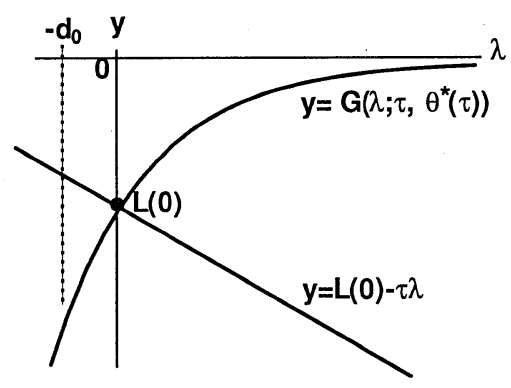

FIG. 3.2. The graphs of $y=G\left(\lambda ; \tau, \theta^{*}(\tau)\right)$ and $y=L(0)-\tau \lambda$ when $L(0)<0$

If $L(0) \leq 0$ (i.e., $\left.\frac{\partial \bar{\Theta}}{\partial \omega}\left(\omega^{*}(\tau)\right) \geq 0\right),(3.13)$ has only the zero solution which comes from the translation invariance. On the other hand, if $L(0)>0$, there exist two solutions. One is the zero and the other is called the critical eigenvalue. We denote this by $\lambda_{c}^{*}\left(\tau, \theta^{*}(\tau)\right)$ (see Fig.3.1 and Fig.3.2). For the sign of the critical eigenvalue, we easily have the following lemma:

LEMMA 3.7.

$$
\operatorname{sgn}\left\{\lambda_{c}^{*}\left(\tau, \theta^{*}(\tau)\right)\right\}=\operatorname{sgn}\left\{-\tau-\frac{d}{d \lambda} G\left(0 ; \tau, \theta^{*}(\tau)\right)\right\}
$$

REMARK 3.8. ([NMIF, Theorem 4.1]) Furthermore we know that

$$
\operatorname{sgn}\left\{-\tau-\frac{d}{d \lambda} G\left(0 ; \tau, \theta^{*}(\tau)\right)\right\}=\operatorname{sgn}\left\{\frac{d}{d \omega} \theta_{O}\left(\omega^{*}(\tau)\right)-\frac{d}{d \omega} \theta_{I}\left(\tau ; \omega^{*}(\tau)\right)\right\} .
$$

Then we know that $\operatorname{sgn}\left\{\lambda_{c}^{*}\left(\tau, \theta^{*}(\tau)\right)\right\}=\operatorname{sgn}\left\{\frac{d}{d \omega} \theta_{O}\left(\omega^{*}(\tau)\right)-\frac{d}{d \omega} \theta_{I}\left(\tau ; \omega^{*}(\tau)\right)\right\}$. Using the technique similar to that in [NMIF], we can also solve the equation (3.12).

Theorem 3.9. For small $\varepsilon>0$, the following holds: (3.12) has only the zero solution when $L(0) \leq 0$. When $L(0)>0,(3.12)$ has two solutions. One is the zero and the other is a critical solution $\lambda(\varepsilon ; \tau, \theta(\tau ; \varepsilon))$, which satisfies $\lim _{\varepsilon \rightarrow 0} \lambda(\varepsilon ; \tau, \theta(\tau ; \varepsilon))=$ $\lambda_{c}^{*}\left(\tau, \theta^{*}(\tau)\right)$ and $\operatorname{sgn}\{\lambda(\varepsilon ; \tau, \theta(\tau ; \varepsilon))\}=\operatorname{sgn}\left\{\frac{d}{d \omega} \theta_{O}\left(\omega^{*}(\tau)\right)-\frac{d}{d \omega} \theta_{I}\left(\tau ; \omega^{*}(\tau)\right)\right\}$. Moreover (3.12) has no other solutions in $\mathbf{C}_{d_{0}}$.

Corollary 3.10. Suppose that $(\mathrm{H} 1),(\mathrm{H} 2),(\mathrm{H} 3)$ and $(\mathrm{H} 4)$ hold. When $\omega_{0} \in I\left(p_{-} \sim p_{+}\right)$in (H4-a) or $\alpha_{13}<\alpha_{23}$ and $\omega_{0} \in I\left(p_{-} \sim p_{+}\right)$in (H4-c), for small $\tau>0\left(\mathbf{u}_{i}, v_{i}\right)(z ; \tau ; \varepsilon)(i=1,3)$ are stable and $\left(\mathbf{u}_{2}, v_{2}\right)(z ; \tau ; \varepsilon)$ is unstable. For other cases in Theorem 2.8, the unique solution is stable for small or large $\tau>0$.

Our numerical example Fig.1.2 corresponds to the case when $\omega_{0} \in I\left(p_{-} \sim p_{+}\right)$in (H4-a) and Fig.1.3 does to the case (H4-b).

4. Concluding Remarks. We considered two-component activator-inhibitor systems in [IMN] and [NMIF] and showed that there always exist three travelling wave solutions, two of them are stable and one is unstable, for small $\tau>0$ when $\omega_{0} \in I\left(p_{-} \sim p_{+}\right)\left(\bar{\Theta}\left(\omega_{0}\right)=0\right)$. But not only the smallness of $\tau$ but also the sign of $\frac{\partial \bar{\Theta}}{\partial \omega}\left(\omega_{0}\right)$ is important for our system. That is, the condition $\frac{\partial \bar{\Theta}}{\partial \omega}\left(\omega_{0}\right)<0$ is necessary 
in the case of $p_{-}<p_{+}$. (Similarly the condition $\frac{\partial \bar{\Theta}}{\partial \omega}\left(\omega_{0}\right)>0$ is necessary in the case of $p_{-}>p_{+}$.) Comparing Fig.2.3 with Fig.2.5, this reason is clarifed mathematically. But we don't know this necessity ecologically. This is a future problem. Indeed, the sign $\frac{\partial \bar{\Theta}}{\partial \omega}\left(\omega_{0}\right)$ is not changeable and always negative in two-component activator-inhibitor systems $[\mathrm{IMN}]$. On the other hand, the positive sign of $\frac{\partial \bar{\Theta}}{\partial \omega}\left(\omega_{0}\right)$ plays interesting phenomena for the same problem in 2-dimensional space. For this, we will show somewhere.

Acknowledgments: The author would like to express his gratitude to Professor Masayasu Mimura for his continuous encouragement. He is partially supported by the Grant-in-Aid for Scientific Research (No.11640107, No.11304005), the Ministry of Education, Science, Sports and Culture, Japan.

\section{REFERENCES}

[I] IKEDA, H., Global bifurcation phenomena of standing pulse solutions for three-component systems with competition and diffusion, Hiroshima Math. J., 32 (2001), pp. 87-124.

[IMN] Ikeda, H., Mimura, M., AND NishiURA, Y., Global bifurcation phenomena of traveling wave solutions for some bistable reaction-diffusion systems, Nonlinear Analysis TMA, 13 (1989), pp. 507-526.

[K] KAN-ON, Y., Parameter dependence of propagation speed of travelling waves for competition-diffusion equations, SIAM J. Math. Anal., 26 (1995), pp. 340-363.

[KY] KAN-ON, Y., AND YANAGIDA, E., Existence of non-constant stable equilibria in competition-diffusion equations, Hiroshima Math. J., 33 (1993), pp. 193-221.

[M1] Miller, P.D., Nonmonotone waves in a three species reaction-diffusion model, Methods and Applications of Analysis, 4 (1997), pp. 261-282.

[M2] Miller, P.D., Stability of non-monotone waves in a three-species reaction-diffusion model, Proceedings of the Royal Society of Edinburgh, 129A (1999), pp. 125-152.

[NF] NishiURA, Y., AND FUJII, H., Stability of singularly perturbed solutions to systems of reaction-diffusion equations, SIAM J. Math. Anal., 18 (1987), pp. 1726-1770.

[NMIF] Nishiura, Y., Mimura, M., Ikeda, H., ANd Fujil, H., Singular limit analysis of stability of traveling wave solutions in bistable reaction-diffusion systems, SIAM J. Math. Anal., 21 (1990), pp. 85-122.

[T] TANIGUCHI, M., A uniform convergence theorem for singular limit eigenvalue problems, submitted. 